\title{
Comparison of neuroglobin distribution and expression in the retina of adult yak and cattle ${ }^{1)}$
}

\author{
XIAOHUA DU, JAMES BLACKAR MAWOLO*, XIAOYU MI*, YANG YANG, XIA LIU*
}

\author{
College of Veterinary Medicine, *College of Life Science and Technology, \\ Gansu Agricultural University, Lanzhou City, Gansu Province, People's Republic of China
}

Du X., Mawolo J. B., Mi X., Yang Y., Liu X.

Comparison of neuroglobin distribution and expression in the retina of adult yak and cattle

\section{Summary}

The yak belongs to the genus Bos and is therefore related to cattle (Bos primigenius species). The yak may have diverged from cattle at any point between one and five million years ago, and the yak is supposed to be more closely related to cattle than to other members of its designated genus. Here, we evaluated the distribution of neuroglobin (NGB) expression in the retina of adult yak and cattle. Five healthy yaks and five cattle were used in the study. Immunohistochemical stainings were performed to assess the distribution of NGB in the retina of adult yak and cattle. The results demonstrated that NGB was expressed at high levels in the retina of adult yak and cattle in the ganglion cell layer, outer plexiform layer, photoreceptor inner segments, and pigment epithelial layer (+++). Medium NGB expression was found in the nerve fiber layer, ganglion cell layer, inner plexiform layer, and photoreceptor outer segments $(++)$. In contrast, NGB was only weakly expressed in the inner nuclear layer $(+)$, while no expression was found in the outer nuclear layer $(-)$. Expression in the inner limiting membrane, outer limiting membrane, and optic nerves of the cattle was weak $(+)$ and comparable to expression in the adult yak. No NGB expression was found in the outer nuclear layer of both yak and cattle. The level of NGB expression in the retinal ganglion cell layer, kernel layer, optic nerve, and photoreceptor inner segments was significantly higher in yak than in cattle $(P<0.05)$. These results suggest that NGB might play an important role in oxygen homeostasis of the retina and normal function of the optic nerve of yak and cattle under high-altitude hypoxic conditions. Nevertheless, its specific functional mechanism needs further investigation.

Keywords: neuroglobin, retina, homeostasis, yak, cattle

Neuroglobin (NGB), first described by Burmester et al. in 2000 (5), is a specific oxygen-binding heme protein, which is found mainly in the brain and retina of vertebrates. NGB stores oxygen and promotes the diffusion of oxygen into mitochondria or directly mediates the transmission of oxygen to mitochondria, promoting the production of adenosine 5'-triphosphate (ATP) and maintaining the normal function of neurons (10). In recent years, studies on NGB have focused on the central nervous system. The internal expression of NGB in the retina and its physiological functions have become a topic of interest for researchers (3). NGB has been detected in the brains of humans, mice, rats, chickens, zebrafish, and pufferfish $(2,4,6,24,32,33$,

\footnotetext{
The researchers extend heartfelt thanks and appreciation to the National Natural Science Foundation of China (Grant No. 31760305) and the Youth Tutor Foundation of Gansu Agricultural University (Grant No. GAU-QNDS-201501) for financial and technical support. This work could not have been possible without assistance rendered by the groups aforementioned. The funders had no role in study design, data collection and analysis, decision to publish, or preparation of the manuscript.
}

$35,46)$, as well as in the eyes of mice, chickens, and zebrafish $(2,21,33,36)$. Vallone et al. found features of NGB in the crystal structure of murine iron storage proteins that are important for the mechanism of ligand binding (32). They further observed that small internal cavities can be found in the heme distal side, which enables the His64(E7) side chain to leave the passageway upon exogenous ligand binding. Meanwhile, studies of the human retina revealed that NGB is generated spontaneously as a result of rapid autoxidation and that it binds exclusively to the GDP-bound form of the alpha subunit of heterotrimeric $G$ proteins $\left(G \alpha_{i}\right)$. NGB expression in the mouse brain, as reported by Wystub et al. (36), shows that NGB is expressed solely in the cerebral cortex of mice and other regions. The estimated concentration of NGB in the retina is about 50 to 100 -fold higher than in the brain, and it is in the same range as that of myoglobin in the muscle (29). The relative oxygen consumption and respiration rate of ocular tissues are uniquely high compared with 
those of the brain and other vertebrate tissues. Retinal energy requirements are such that this tissue has the highest rate of oxygen consumption in the body (1, $27,34,39)$, suggesting that NGB may be linked to the use of oxygen by the retina. The yak (Bos grunniens), a type of ruminant, differentiated from other ruminants about 1-5 million years ago. It lives and breeds exclusively on the Qinghai-Tibetan Plateau, China, at 3000 $\mathrm{m}$ above sea level in a cold mountainous area. It has excellent adaptation to high-altitude hypoxia. Because NGB plays an extremely important role in the uptake, transportation, and utilization of oxygen, it is hypothesized that it plays an important role in the adaptation of yak to the hypoxic environment of high altitudes. The exact function of NGB in hypoxia or ischemia remains a matter of debate (27). Several studies on NGB have recently been conducted on the retinas of rodents, dogs, humans, rabbits, and sheep $(18,22,23$, 37, 40). Ilmjarv et al. (2014) showed in their study on the mouse retina that the lack of NGB has only a small effect on the light-induced retinal gene expression response (16). A report demonstrated that the genetic elimination of NGB does not affect the core clock function, but evokes an increased behavioral response to light concomitant with increased Per1 expression in the subthalamic nucleus at early night (15). Another study reported that NGB might participate in cellular defense against hypoxia (9). The study further showed that an important likely function of NGB is to scavenge reactive oxygen and nitrogen species that are generated following brain hypoxia. Meanwhile, Fabrizius et al. (2016) found a low embryonic expression of $\mathrm{Ngb}$ mRNA, which increased after birth, indicating a role of this protein in fetal hypoxia tolerance. In addition, the highest expression of Ngb mRNA was observed in the hypothalamus, where it was up to 100-fold higher than in the cerebral cortex, cerebellum, or hippocampus (8). However, no information is available comparing the distribution and expression of NGB in the retina of adult yak and cattle. Therefore, the present study was designed to compare the distribution of NGB and its expression in the retina of adult yak and cattle via immunohistochemistry. These current results provide valuable morphological data for further investigations of the distribution of NGB in the mammalian retina and a basis for studying physiological functions of NGB in retinal hypoxic injury-related diseases.

\section{Material and methods}

Animals and setting. Five healthy adult yaks aged five years were included in this study. Cattle and yak retina samples were purchased from Gannan Tibetan Autonomous Prefecture in Gansu Province, China. This area is a plateau environment at an altitude of $3000 \mathrm{~m}$. Another five healthy adult cattle aged five years were involved in this research and were purchased from Pinliang in Gansu Province, China. This region is a plain environment at an altitude of 1500 meters.
Reagents and devices. The following reagents were purchased and used in this study: phosphate buffer solution (PBS) ZLI-9062, an immunohistochemical staining kit SP-9001, and a DAB color reagent kit ZLI-9018, all from Beijing Zhongshan Golden Bridge Biotechnology Co., Ltd, China. Trypsin (T8150-25) was obtained from Beijing Solarbio Technology Co., Ltd. P.R. China, and formaldehyde and N-butanol solution (analytically pure) were acquired from Tianjin Baishi Chemical Co., Ltd. China. Additional reagents used in this study were glacial acetic acid and alcohol solution (analytically pure) from Tianjin Fuyu Fine Chemical Co., Ltd. China and rabbit anti-mouse polyclonal antibody (BS-1859R) from Beijing Boaosen Biotechnology Co., Ltd. China.

Conventional surgical instruments were used for the extraction of the eyes: scalpels, anatomical scissors, KD-BM, KD-P, and KD-H from Zhejiang Jinhua Kadi Instrument Equipment Co., Ltd. P.R. China, DHP-9082 from Shanghai Duofu Industry Co., Ltd. P.R. China, and RM-2235 and 13395H2X from Leica, Germany. A microtome was used to cut the tissue sections.

Treatment and specimen sampling (immunohistochemistry). Experiments were carried out using yak and cattle weighing $350-585 \mathrm{~kg}$ and $550-720 \mathrm{~kg}$, respectively. The weight of each retina section from cattle and yak was determined and recorded. The weight ranged from 10-15 fold. The animals were kept at a temperature between $7^{\circ} \mathrm{C}$ and $-8^{\circ} \mathrm{C}$ and had ad libitum access to food and water. Experimental procedures were conducted in accordance with the Animal Ethics and Welfare Committee of Gansu Agricultural University. Every effort was made to reduce the number of animals used and to minimize animal suffering during the experiment. Samples were taken by trained personnel from the cornea to extract the lens and the vitreous body. Animals were retrieved one at a time from their living areas and minimally immobilized to facilitate sacrifice and the extraction of the eyes. The research center required minimum immobilization of the animals to reduce complications. Therefore, we followed this guidance of the resident veterinarians to reduce harm and pain to the animal.

Upon sacrificing the animals, the eyes were quickly removed and placed in Davidson's fixative $(95 \%$ alcohol, formaldehyde, glacial acetic acid, and distilled water; the ratio of $3: 2: 1: 2$ ) for 24 hours. Sharp blades were used to open the cornea and extract the lens and the vitreous body. The fixed eye tissue was flushed for five hours under running water and kept in 70\% alcohol (overnight), 80\% alcohol (five hours), 90\% alcohol (three hours), 95\% alcohol (two hours), and N-butanol (overnight). Afterwards, the eyes were placed in a new N-butanol solution for five hours (28) and then embedded in paraffin to generate paraffin sections.

Immunohistochemical staining procedures were used. The steps were as follows: paraffin sections were dewaxed via gradient ethanol hydration. The sections were rinsed three times (five minutes each) in PBS $(0.01 \mathrm{~mol} / \mathrm{L}, \mathrm{pH}=7.2)$, treated with 0.125 trypsin antigen repair for 30 minutes, and then rinsed in PBS. Each slice was incubated for ten minutes at $20^{\circ} \mathrm{C}$ room temperature with a drop of $30 \mathrm{~mL} / \mathrm{L}$ hydrogen peroxide blocking solution and then rinsed in PBS. Normal goat serum was added and the sections were 
incubated at room temperature for 15 minutes. One drop of the corresponding primary antibody was added, and the sections were incubated at $37^{\circ} \mathrm{C}$ for two hours, followed by three washes with PBS. Then, PBS was removed, and the corresponding second antibody was added for 15 minutes at $37^{\circ} \mathrm{C}$ before rinsing the sections three times with PBS and subsequent immersion in PBS. A drop of Streptomyces biotin-peroxidase solution was added to each slice. This was incubated at $37^{\circ} \mathrm{C}$ for 15 minutes before rinsing the sections with PBS. PBS was then removed and DAB mixed with PBS was added. The color was observed and controlled under a microscope. Distilled water was added to terminate the staining. The sections were then redyed with hematoxylin, dehydrated, encapsulated with transparent and neutral gum, photographed, and finally analyzed under a microscope. The negative control sections were stained with PBS without the primary antibody.

Determination of results. Uniform parameters were chosen for recording the images on an Olympus DP71 micro camera system. The Image-Pro Plus 6.0 software was used to analyze the images. Both the area and the integrated optical density in the targeted image area were measured within the measurement window. The measurement results were imported into the Excel 2010 software to calculate the mean density (MD) of each layer (MD integrated optical density/area). The MD of the negative control group was measured similarly as described above. The MD value of each layer structure area in the negative control group was 0 . It was considered as no expression $(-)$, and where a significant difference was present, it was referred to as a positive expression. Positive MD values were divided into three categories: low $(+)$, medium $(++)$, and high $(+++)$ expression. $(-)$ indicates no expression.

Ethics statement. The experimental protocols were approved by the Animal Ethics and Welfare Committee of Gansu Agricultural University, Gansu Province in October 2018 with regard to the protection of animals used in research and for scientific purposes under approval no. AEWC-GAU-2018019.

Anesthesia and efforts to alleviate suffering. In accordance with the regulations of the Animal Ethics and Welfare Committee of Gansu Agricultural University, all animals involved in the study were placed separately until they were confirmed to be healthy. The animals were observed for two weeks before further procedures were performed according to the committee regulations. The animals were free while under observation, and the observation confirmed that they were free of specified infectious diseases that could have an adverse effect on the experimental procedures. The animals' feed was provided to them while under observation, and no search for food, chewing, grubbing, and gnawing during the activity periods. To avoid or minimize pain, the animals were handled calmly by trained personnel. Dealing with these large animals requires more personnel, so additional trained personnel were employed for assistance during the sacrifice. The animals were spoken to by the personnel, and loud sounds were avoided so as not to frighten them. At the same time, food was given to the animals so that they could interact and develop a relationship with the personnel. The animals were made to lie on their side by scratching their back and flanks by the personnel. When the animals were calm, the injections were administrated and the eyes were extracted. The injections were performed under slow pressure to reduce pain, and then the animal tissues and other organelles were carefully extracted. The injection needles were appropriate for the animal size. The environment was well lit, and sharp or damaged objects had been removed.

Laboratory protocols. The procedures used in this research were deposited at protocols.io and can be found at DOI: dx.doi.org/10.17504/protocols.io.bb3hiqj6.

Statistical analysis. The MD results are expressed as mean $\pm \mathrm{SD}(\overline{\mathrm{x}} \pm \mathrm{s})$, and the expression intensity as described above. The MD values of the retinal layers and negative controls were analyzed by the SPSS 18.0 statistical software. A one-way analysis of variance was employed to evaluate the data of yak and cattle retinal layers. A t-test was employed to test whether the differences between yak and cattle MD values were significant $(\mathrm{P}<0.05)$.

\section{Results and discussion}

Anatomic structure of the retina of adult yak and cattle. The retina is a thin layer of tissue that lines the inside of the back of the eye. It is a transparent membrane located near the optic nerve. Yak and cattle retinas, like those from most known mammals (18), can be divided into ten layers. The results of the statistical analysis showed that NGB was highly expressed in the ganglion cell layer, photoreceptor inner segments, and pigment epithelial layer $(+++)$, while expression levels in the outer plexiform layer, nerve fiber layer, inner plexiform layer, and photoreceptor outer segments were medium. NGB was only weakly expressed in the inner nuclear layer $(+)$, while no expression was found in the outer nuclear layer $(-)$ (Tab. 1). The results for the cattle retina showed that NGB expression intensity in the experimental group was high $(+++)$ in the outer plexiform layer, photoreceptor inner segments, and pigmented epithelial layer, while in the nerve fiber layer, ganglion cell layer, inner plexiform layer, and photoreceptor outer segments, NGB expression was

Tab. 1. Neuroglobin expression intensity in the adult yak retina $(\overline{\mathbf{x}} \pm \mathbf{s})$

\begin{tabular}{|l|c|c|}
\hline \multicolumn{1}{|c|}{ Layer } & Mean density & $\begin{array}{c}\text { Expression } \\
\text { intensity }\end{array}$ \\
\hline Nerve fiber layer & $0.112 \pm 0.019 * * *$ & ++ \\
\hline Ganglion cell layer & $0.153 \pm 0.030^{* * * \Delta}$ & +++ \\
\hline Inner plexiform layer & $0.118 \pm 0.017^{* * *}$ & ++ \\
\hline Inner nuclear layer & $0.089 \pm 0.009^{* *}$ & + \\
\hline Outer plexiform layer & $0.144 \pm 0.017^{* * *}$ & ++ \\
\hline Outer nuclear layer & $0.025 \pm 0.007^{*}$ & - \\
\hline Photoreceptor inner segments & $0.185 \pm 0.010^{* * * \Delta}$ & +++ \\
\hline Photoreceptor outer segments & $0.126 \pm 0.022^{* *}$ & ++ \\
\hline Pigmented epithelial layer & $0.177 \pm 0.047^{* * * \Delta}$ & +++ \\
\hline
\end{tabular}

Explanations: * - compared with the negative control group, $\mathrm{P}>0.05 ; * *$ - compared with the negative control group, $\mathrm{P}<0.05$;

$\Delta$ - intra-group comparisons of the experimental group, $\mathrm{P}<0.05$ 
medium $(++)$. NGB expression intensity in the inner nuclear layer was weak $(+)$. The outer nuclear layer had no NGB expression (Tab. 2).

Tab. 2. Comparison of neuroglobin expression intensity in the adult cattle retina $(\bar{x} \pm s)$

\begin{tabular}{|l|c|c|}
\hline \multicolumn{1}{|c|}{ Layer } & Mean density & $\begin{array}{c}\text { Expression } \\
\text { intensity }\end{array}$ \\
\hline Nerve fiber layer & $0.100 \pm 0.026^{* * *}$ & ++ \\
\hline Ganglion cell layer & $0.104 \pm 0.010^{* * *}$ & ++ \\
\hline Inner plexiform layer & $0.105 \pm 0.014^{* * *}$ & ++ \\
Inner nuclear layer & $0.067 \pm 0.010^{* *}$ & + \\
\hline Outer plexiform layer & $0.137 \pm 0.013^{* * *}$ & ++ \\
\hline Outer nuclear layer & $0.019 \pm 0.005^{*}$ & - \\
\hline Photoreceptor inner segments & $0.131 \pm 0.019^{* * *}$ & ++ \\
\hline Photoreceptor outer segments & $0.106 \pm 0.021^{* *}$ & ++ \\
\hline Pigmented epithelial layer & $0.159 \pm 0.038^{* * * \Delta}$ & +++ \\
\hline
\end{tabular}

Explanations: as in Tab. 1.
Immunohistochemistry and semi-quantitative analysis of NGB expression in the retina of adult cattle. The results from the immunohistochemical analysis of adult cattle were similar to those for adult yak. NGB immunoreactive substances were distributed across all layers, with the expression intensity being significantly higher in all layers other than the inner nuclear layer, in which it was lower than in the other regions (Fig. 1-2).

Comparison of NGB expression and distribution in the retina of yak and cattle. The qualitative analysis revealed NGB expression in yak and cattle retinas in all layers except the outer nuclear layer. An Intel integrated performance primitive (IPP) comparative analysis of the expression of NGB in yak and cattle retinas revealed that protein expression was high $(+++)$ in the ganglion cell layer, photoreceptor inner segments, pigment epithelial layer, outer plexiform layer, and photoreceptor inner segments of yak and
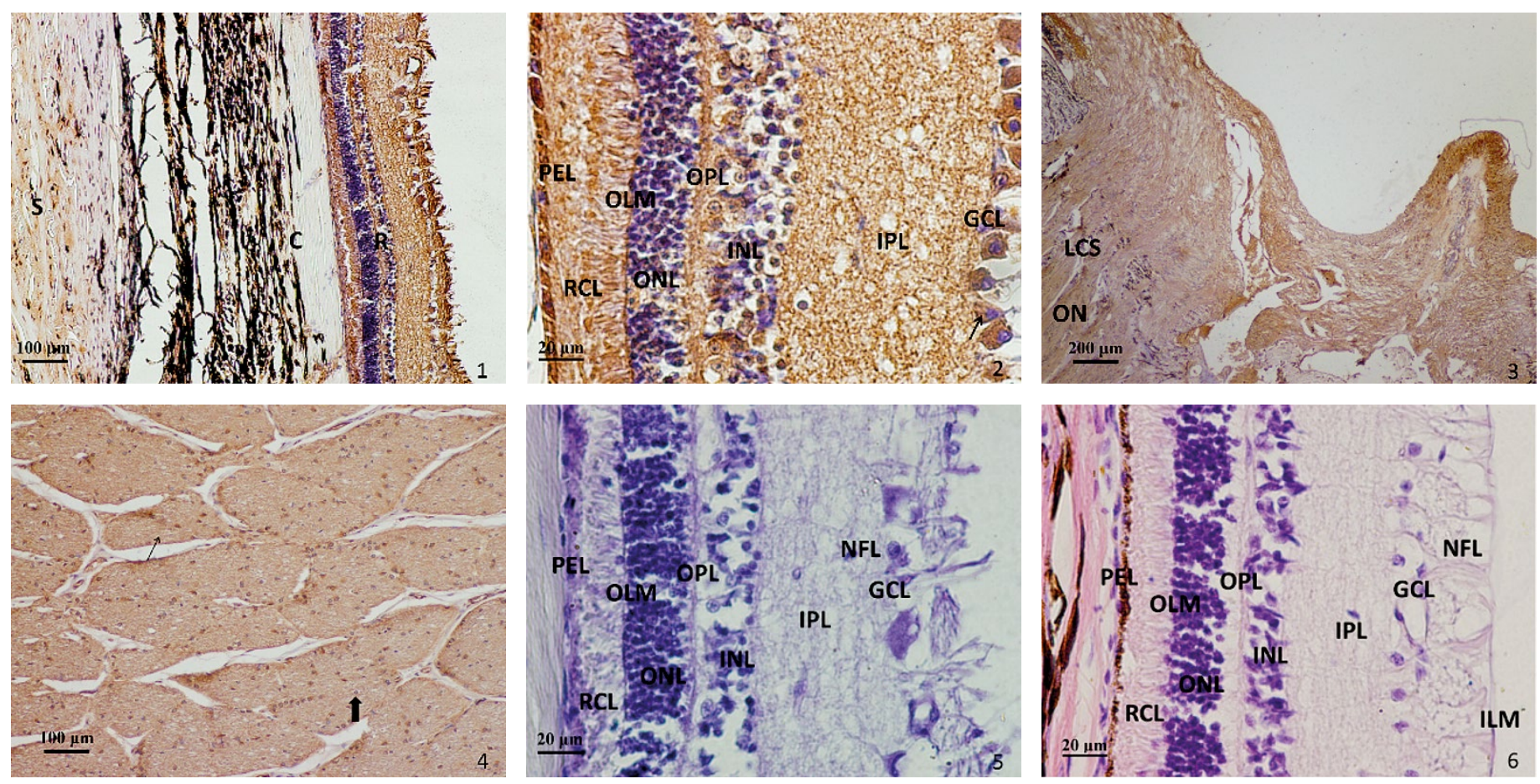

Fig. 1-1. NGB expression in the eyeball wall of adult yak and cattle. Immunohistochemical staining, scale bar $=100 \mu \mathrm{m}$. $R$ - retina (10 $\mu \mathrm{m}$, width); $C$ - choroid $(12 \mu \mathrm{m}) ; S$ - sclera $(10 \mu \mathrm{m})$

Fig. 1-2. NGB expression in the retina of adult yak and cattle. Immunohistochemical staining, scale bar $=100 \mu \mathrm{m}$. NFL nerve fiber layer $(10 \mu \mathrm{m})$; GCL - ganglion cell layer $(13 \mu \mathrm{m})$; IPL - inner plexiform layer $(10 \mu \mathrm{m})$; INL - inner nuclear layer $(10 \mu \mathrm{m}) ; \mathrm{OPL}$ - outer plexiform layer $(14 \mu \mathrm{m}) ; \mathrm{ONL}$ - outer nuclear layer $(15 \mu \mathrm{m}) ; \mathrm{RCL}$ - a layer of rods and cones $(15 \mu \mathrm{m})$; PEL - pigmented epithelial layer $(14 \mu \mathrm{m}) ; \uparrow$ highlights the retinal ganglion cells

Fig. 1-3. NGB expression in the optic disc of adult yak and cattle. Immunohistochemical staining, scale bar $=200 \mu \mathrm{m}$. LCS - lamina cribrosa sclerae $(10 \mu \mathrm{m}) ; \mathrm{ON}$ - optic nerve $(10 \mu \mathrm{m})$

Fig. 1-4. NGB expression in the optic nerve of adult yak and cattle. Immunohistochemical staining, scale bar $=100 \mu \mathrm{m}$. $\uparrow$ highlights the fascicle of optic nerve fibers $(10 \mu \mathrm{m})$; 1 highlights the glial cells

Fig. 1-5. NGB expression in the optic disc of adult yak and cattle. Immunohistochemical staining, scale bar $=200 \mu \mathrm{m}$. ILM - inner limiting membrane $(10 \mu \mathrm{m})$; NFL - nerve fiber layer $(10 \mu \mathrm{m})$; GCL - ganglion cell layer $(11 \mu \mathrm{m})$; IPL - inner plexiform layer $(13 \mu \mathrm{m})$; INL - inner nuclear layer $(15 \mu \mathrm{m})$; OPL - outer plexiform layer (14 $\mu \mathrm{m})$; ONL - outer nuclear layer $(10 \mu \mathrm{m}) ;$ OLM - outer limiting membrane $(10 \mu \mathrm{m})$; RCL - a layer of rods and cones $(15 \mu \mathrm{m})$; PEL - pigmented epithelial layer $(12 \mu \mathrm{m})$

Fig. 1-6. The retina of adult yak and cattle, H-E staining, scale bar $=200 \mu \mathrm{m}$. ILM - inner limiting membrane (15 $\mu \mathrm{m})$; NFL - indicates nerve fiber layer $(10 \mu \mathrm{m})$; GCL - ganglion cell layer $(12 \mu \mathrm{m})$; IPL - inner plexiform layer $(10 \mu \mathrm{m})$; INL - inner nuclear layer (15 $\mu \mathrm{m})$; OPL - outer plexiform layer $(15 \mu \mathrm{m})$; ONL - outer nuclear layer (14 $\mu \mathrm{m})$; OLM - outer limiting membrane $(15 \mu \mathrm{m}) ; \mathrm{RCL}-$ a layer of rods and cones $(12 \mu \mathrm{m}) ;$ PEL - pigmented epithelial layer $(15 \mu \mathrm{m})$ 
cattle. Both yak and cattle retinas showed medium $(++)$ NGB expression in the outer plexiform layer, nerve fiber layer, inner plexiform layer, photoreceptor outer segments, ganglion cell layer, and photoreceptor outer segments. Weak NGB expression was observed in the inner nuclear layer, and no NGB expression was found in the outer nuclear layer of either yak and cattle retinas. NGB expression in the ganglion cell layer was high in the yak retina $(+++)$ and medium $(++)$ in the cattle retina. NGB expression in the outer plexiform layer was medium $(++)$ in the yak retina and high $(+++)$ in the cattle retina. The statistical results for NGB MD showed that the expression of NGB in the retinal ganglion cell layer, inner nuclear layer, and photoreceptor inner segments in yak was significantly higher than in cattle $(\mathrm{P}<0.05)$. The nerve fiber layer, photoreceptor outer segment, and inner plexiform layer in yak and cattle retinas showed similar levels of NGB expression $(\mathrm{P}>0.05)$. In addition, the expression of NGB in the optic nerve of yak was significantly higher than that in cattle $(\mathrm{P}<0.05)$. Therefore, the overall level of NGB expression in the retina was higher in yak compared to cattle.

Similar to previous studies on the retina of rodents, dogs, and humans, we found NGB in the ganglion cell layer, inner plexiform layer, outer plexiform layer, and photoreceptor inner segments in adult yak and cattle retinas $(22-24,32,37,40)$. We also showed that NGB was expressed in the nerve fiber layer, inner nuclear layer, outer limiting membrane, and photoreceptor outer segments of both adult yak and cattle, which has not been previously reported. Using immunohistochemistry, Bentmann (3), Yaoyu (40), and Schmidt et al. (29) showed that NGB was not found in the pigment epithelium layer of rats and mice. In contrast, Ostojic $(22,23)$ and Guiling et al. (11) located NGB by the same method in the retinal pigment epithelium layer of humans, canines, and Bactrian camels. Our study has shown that NGB was expressed in the pigment epithelium layer of adult yak and cattle retinas, which is consistent with the results of Ostojic (22, 23) and Guiling et al. (11), but differs from the results of Bentmann et al. (3). These differences may result from diversity in animal species tested, different living environments, the quality of the antibodies, and different specificities of immunological detection. In addition, the inner retina of humans and most mammals is nourished by the central vascular system of the retina, while the blood supply to the outer retina is mainly supported by the choroidal circulation. Since the blood of the choroidal circulation does not reach the photoreceptor cell layer directly, oxygen must pass through the pigment epithelium layer to diffuse to photoreceptors, potentially explaining the difference in NGB expression. Our results on the expression of NGB in the outer nuclear layer of the mammalian retina are inconsistent with earlier studies. Jianhua et al. (18) used in situ hybridization to find strong signals of NGB mRNA hybridization in the inner and outer nuclear layers of the rat retina. This was consistent with the results of Yaoyu (40) and Ostojic et al. (22), who used immunohistochemistry to detect NGB in the eye nucleus of rats and humans. However, studying the subcellular distribution of NGB, Qiong et al. (25) found no NGB expression in the outer nuclear layer. These results are similar to those from our study, but differ from the results of Ostojic et al. (23) and Yaoyu (40). This difference may be related to the different animal species and the specificity of their immune response.

Previously, studies have been conducted to determine the distribution of NGB in the retina of mammals, but none have reported the expression of NGB in the photoreceptor outer segment in the retina. We showed here that NGB was expressed in the photoreceptor outer segment of the retina of adult yak and cattle. These findings may be due to differences in animal species, their adaptability to the environment, and/ or the closely related functions in the visual system of yak and cattle living in plateau and sub plateau environments, respectively. The adaptability of these species to the environment needs further investigation. High-altitude environments pose several unique physiological challenges to the survival of mammals. In addition to low temperatures, high-altitude environments are also characterized by low partial oxygen pressure $\left(\mathrm{PO}_{2}\right)$ relative to low-altitude environments at similar latitudes. Since the reduced $\mathrm{PO}_{2}$ of inspired air will typically result in a reduced $\mathrm{O}_{2}$ saturation of arterial blood, compensatory physiological adjustments may be required to ensure an adequate supply of $\mathrm{O}_{2}$ to the cells of aerobically metabolizing tissues.

The semi-quantitative IPP analysis results have shown that the highest expression of NGB in yak was found in the retinal ganglion cell layer, photoreceptor inner segments, and pigment epithelial layer, among others, while the highest expression in cattle was observed in the outer plexiform layer, photoreceptor inner segments, and pigment epithelial layer. The high expression of NGB in the photoreceptor inner segments of yak and cattle retinas is consistent with the study of Schmidt et al. (29), who studied NGB expression in the mouse retina. Some studies have shown that the ganglion cell layer, the inner plexiform layer, the outer plexiform layer, and the photoreceptor inner segments have the highest oxygen consumption rates in the mammalian retina (25). These regions contain many synaptic connections between two layers of adjacent neurons. As the function of these neurons is to transmit information through these synapses, a large amount of oxygen is consumed. NGB has also been found to be expressed mainly in these sites $(1,7,18,20,23,27,38$, $40,42)$, and its high expression may be closely related to its use of oxygen, and the subcellular location of mitochondria. In addition, the oxygen carried by NGB can also be used by retinal neurons to satisfy the high demand for oxygen throughout the four layers of the 
retina. Schmidt et al, who used electron microscopy to study the retina, found NGB located around the mitochondria, but not inside them. Using the yeast two-hybrid method, Yu et al. (43) found that a subunitcytochrome $(\mathrm{C} 1)$ of mitochondrial complex III can bind to NGB. These studies have had a great influence on further exploration of the mechanisms underlying the physiological functions of NGB. In addition, Qiong et al. found NGB between synaptic vesicles of the presynaptic structure in the retina of adult rats, further supporting the hypothesis that NGB is associated with oxygen consumption in the retina (25). Based on these results, it was also suggested that NGB may be secreted by neurons and transported between synaptic vesicles to perform complex physiological functions. We also found high NGB levels in the pigment epithelium layer of the retina of yak and cattle, compared with other regions. Other studies have shown that the pleural folds of pigment epithelium cells can increase the contact area between pigment epithelial cells and the choriocapillaris. The choriocapillaris favors the selective transport of nutrients and metabolites between the outer retina and the choroid (26). Due to the complex functions of pigmented epithelial cells, they have higher energy requirements, suggesting that the high expression of NGB in the pigment epithelium layer may be related to the need for more oxygen to maintain normal functional activity. In addition, Hongjun (14), who cultured cattle pigment epithelial cells in vitro, found that under hypoxic conditions, the pigment epithelial cell count was significantly higher than that under normal conditions. This suggests that hypoxia could promote the proliferation of pigment epithelial cells. The present study indicates that the high intensity of NGB expression in the pigment epithelium layer of the yak retina is related to its proliferation in retinal pigment epithelial cells under hypoxic conditions. However, this requires further investigation.

Previous studies have shown that NGB maintains the dynamics of retinal oxygen metabolism and that a greater expression of NGB can be maintained over a long period in retinal ischemia and hypoxia (12). Shaoyang et al. (30) found that NGB expression increases rapidly after retinal ischemia, which indicates that NGB is very sensitive to hypoxia and suggests that it may play a protective role in the adaptive process of retinal hypoxia. Guopeng et al. (12) showed that NGB expression in the retina of rats under acute ischemic and hypoxic conditions is found mainly in the ganglion cell layer, and some expression is also found in the inner and outer plexiform layers. Additionally, Kayo et al. (19) found that in the retina of control mice, NGB immunoreactivity was limited to the retinal ganglion cell layer. It has been suggested that NGB, which is found in high concentrations within the retina, may help to maintain an adequate supply of oxygen via oxygen transport and storage. Roberts et al. (28) modeled this mathematically and demonstrated that the retina is most susceptible to hypoxia in the regions of the photoreceptor inner segments and inner plexiform layers. In these layers, NGB has the potential to prevent hypoxia and increase oxygen uptake by $30-40 \%$. Zhenguo et al. (44) showed that, compared to plain and sub-plateau environments, a high-altitude hypoxic environment increases the expression of NGB. Shufen et al. (31) showed that the increased expression of NGB during acute and chronic exposure to high-altitude hypoxic environments enhances the tolerance of rats to hypoxia. These results suggest that the change in NGB expression may be an endogenous neuroprotective response of the brain tissue to cerebral hypoxia aimed at increasing oxygen supply to the brain tissue and maintaining the normal neuronal function. In the present study, NGB expression in the retinal ganglion cell layer, inner nuclear layer, and photoreceptor inner segments was significantly higher in yak than in cattle. Ying (41) showed that transient global ischemia induces a dysfunction of retinal ganglion cells and amacrine cells and a transient change in bipolar cells, but does not affect photoreceptors. It was suggested that the high expression of NGB in the retina of yak in a high-altitude hypoxia environment enhances retinal oxygen utilization and maintains the normal physiological function of the retina.

In this study, NGB expression in the retina of yak living in a high-altitude environment showed that NGB has the highest expression intensity in the ganglion cell layer, photoreceptor inner segments, and pigment epithelial layer. NGB was highly expressed in the nerve fiber layer, inner plexiform layer, and photoreceptor outer segment of the yak retina, while weak NGB expression was observed in the inner nuclear layer. The high expression of NGB in the retina of cattle living in a high-altitude environment demonstrated that the sub-plateau environment influenced the level of NGB expression. These results suggest that the differences in NGB expression in various layers of the retina may also be related to the sensitivity of each layer to hypoxia-ischemia injury. The areas of the yak and cattle retina that have the highest oxygen consumption, the photoreceptor inner segments and the pigment epithelium layer, are the most sensitive to hypoxia-ischemia. The yak ganglion cell layer of the retina is the most sensitive to ischemia and hypoxia. The ganglion cell layer serves as a pathway for retinal information that passes into the brain and consumes large amounts of oxygen. The outer plexiform layer is one of the most oxygen-consuming parts of the retina. The pigmented epithelial cells that are actively metabolized show special phagocytosis and are responsible for the biological filtration function of the retina neurosensory layer, requiring a higher energy supply. Therefore, cells in this layer have the highest sensitivity to ischemia and hypoxia. In yak and cattle, the retinal optic nerve fiber layer, inner plexiform layer, photoreceptor outer segment, and other areas, which are the adjacent layers of 
synaptic contacts between the neurons and the pathway for retinal information into the brain, also have a high oxygen demand. They are highly sensitive to ischemia and hypoxia. The ganglion cell layer of the cattle retina and the outer plexiform layer of the yak retina are also highly sensitive to ischemia and hypoxia. In addition, the inner nuclear layer serves as a pathway for photoreceptor cell signals to the ganglion cells, which are also highly sensitive to ischemia and hypoxia. The site without NGB expression in the outer nuclear layer was observed to be the least sensitive to hypoxia.

In this study, high NGB expression was observed in the yak and cattle optic nerve, which is consistent with the results of Yaoyu L's studies on rats, suggesting that NGB is involved in the normal function of the optic nerve of yak and cattle. A large number of studies have shown that the high NGB expression in the central nervous system has a protective effect against ischemic and hypoxic damage to the central nervous system $(14,15,46)$. The present study suggests that the overall intensity of NGB immunoreactivity in the retina is higher in yak than it is cattle. Therefore, NGB may play an important physiological role in the normal functioning of the optic nerve in yak under high-altitude hypoxic conditions. However, its specific functional mechanism requires further studies.

\section{References}

1. Anderson B.: Ocular effects of changes in oxygen and carbon dioxide tension. Trans. Am. Ophthalmol. Soc.1968, 1, 423-474.

2. Becquet F., Courtois Y., Goureau O.: Nitric oxide in the eye: multifaceted roles and diverse outcomes. Surv. of Ophthalmol. 1997, 42, 71-82.

3. Bentmann A., Schmidt M., Reuss S., Uwe W., Thomas H.: Divergent distribution in vascular and avascular mammalian retinae links neuroglobin to cellular respiration. J. Histochem. Cytochem. 2005, 50, 1591-1598.

4. Bosch-Morell F., Româ J., Marin N., Belén R., Antonio R. G.: Role of oxygen and nitrogen species in experimental uveitis: anti-inflammatory activity of the synthetic antioxidant ebselen. Free Radic. Biol. Med. 2002, 33, 669-675.

5. Burmester T., Weich B., Reinhardt S.: A vertebrate globin expressed in the brain. Nature. 2000, 6803, 520-523, doi: 10.1038/35035093.

6. Cejkovâ J., Stipek S., Crkovskâ J., Ardan T., Plátenik J.: UV Rays, the pro-oxidant/antioxidant imbalance in the cornea and oxidative eye damage. Physiol. J. 2004, 53, 1-10.

7. Cringle S. J., Yu D. Y., Paula K. Y.: Intra-retinal Oxygen Consumption in the Rat In Vivo. Investig. Ophthalmol. Vis. Sci. 2002, 6, 1922-1927.

8. Fabrizius A., Andre D., Laufs T, Bicker A., Reuss S.: Critical re-evaluation of neuroglobin expression reveals conserved patterns among mammals. Neuroscience 2016, 337, 339-354.

9. Fago A., Hundahl C., Malte H., Roy E. W.: Functional properties of neuroglobin and cytoglobin. Insights into the ancestral physiological roles of globins. Iubmb Life 2004, 56, 689-696.

10. Geuens E., Brouns I., Flamez D., Sylvia D., Jean-Pierre T.: A globin in the nucleus. Journal of Biological Chemistry 2003, 33, 30417-30420, doi: 10.1074 jbc.C300203200

11. Guiling H., Xia L., Xiaona H.: Expression of neuroglobin in the retina of the Bactrian camel. Chi. Vet. Sci. 2018, 48, 354-359.

12. Guopeng D., Zhenguo Y., Tao H.: Expression of neuroglobin during cell death in rat acute retinal ischemia. Chi. J. Ophthalmol. 2010, 7, 590-596.

13. Hao Z., Yajun L.: Neuroprotective effect of neuroglobin and its possible mechanism. IJNNS 2017, 44, 668-671.

14. Hongjun D., Shouyi G.: Effect of Hypoxia on Proliferation of Bovine Retina Pigment Epithelial Cells. Depart. Ophthalmol. 1997, 4, 239-240.

15. Hundahl C. A., Fahrenkrug J., Hay-Schmidt A., Anders H. S., Birgitte G. Circadian behavior in neuroglobin deficient mice. Plos One 2012, 4, doi: 10.1371/journal.pone.0034462.

16. Ilmjärv S., Reimets R., Hundahl C. A., Hendrik L.: Effect of light on global gene expression in the neuroglobin-deficient mouse retina. Biomed. Rep. 2014, $26,780-786$
17. Ostojić J.: Neuroglobin and cytoglobin distribution patterns in the human and canine eye. Biol. Chem. 2006, 21, 20660-20665.

18. Jianhua R., Shanquan S., Yan B.: Expression of neuroglobin mRNA in the eye of rats. Neurosc. 2005, 7, 1035-1036, doi: 10.1016/s0306-4522(02)00536-5.

19. Kayo S., Yoshiki K., Mayuko S., Kunizo A., Kazuhiro O.: A novel function of neuroglobin for neuro regeneration in mice after optic nerve injury. Biochem. Biophys. Res. Commun. 2017, 493, 1254-1259.

20. $\mathrm{Li}$ K.: Expression and clinical significance of neuroglobin in eyes and serum of patients with proliferative diabetic retinopathy. Sha. Med. Uni. 2016, 12 112-123.

21. Mammen P. P., Shelton J. M., Goetsch S. C., Williams S. C., Richardson J. A.: Neuroglobin, a novel member of the globin family, is expressed in focal regions of the brain. J. Histochem. Cytochem. 2002, 8, 89-97.

22. Ostojić J., Grozdanić S. D., Syed N. A., Hargrove M. S., Trent J. T.: Patterns of distribution of oxygen-binding globins, neuroglobin, and cytoglobin in the human retina. Arch. Ophthalmol. 2008, 11, 1530-1536.

23. Ostojić J., Sakaguchi D. S., De Lathouder Y., Hargrove M. S., Trent J. T. Neuroglobin and cytoglobin: oxygen-binding proteins in retinal neurons. Investig. Ophthalmol. Vis. Sci. 2006, 43, 1016-1023.

24. Parker N. R., Jamie J. F., Davies M. J., Truscott R. J.: Protein-bound kynurenine is a photosensitizer of oxidative damage. Free Radic. Biol. Med. 2004, 37 1479-1489.

25. Qiong X., Xiaofeng H., Xiaoyan W., Xiao-Long C.: Subcellular distribution of neuroglobin in adult rat retina. IJO 2009, 91, 35-37.

26. Qiuming L., Guangyi Z.: Applied anatomy of ophthalmology [M]. Zheng. Uni 2013, 5, 121-124.

27. Rajendram R., Rao N. A.: Neuroglobin in normal retina and retina from eyes with advanced glaucoma. Br. J. Ophthalmol. 2007, 915, 663-666.

28. Roberts P. A., Gaffney E. A., Philip L. J., Alexander J. E. F., Helen M. B. Retinal oxygen distribution and the role of neuroglobin. J. Math. Biol. 2016, 73, 1-38

29. Schmidt M., Giessl A., Laufs T., Thomas H., Uwe W.: How does the eye breathe? Evidence for neuroglobin-mediated oxygen supply in the mammalian retina. J. Biol. 2003, 278, 1932-1935.

30. Shaoyang S., Xuemei F.: Expression of Neuroglobin in Rat Acute Retinal Ischemia Injury. Depart. of Ophthalmol. 2009, 2710, 915-918 (in Chinese).

31. Shufen H., Zhenzhong B., Yue C.: High-Altitude Hypoxia Increased Neuroglobin Expression in Rat Cerebral Cortices. Chin. Med. J. 2017, 38, 73-80.

32. Vallone B., Nienhaus K., Brunori M., Nienhaus G. U.: The structure of murine neuroglobin: Novel pathways for ligand migration and binding. Proteins 2004 $56,85-92$.

33. Wakasugi K., Nakano T., Morishima I.: Oxidized human neuroglobin acts as a heterotrimeric Galpha protein guanine nucleotide dissociation inhibitor J. Biol. 2003, 278, 36505-36512.

34. Wei X., Deng Y., Liu X.: How can photoreceptor cells breathe in the retina? Neuroglobin may be the answer. Neural Regen. Res. 2010, 59, 720-731.

35. Wu G. S., Zhang J., Rao N. A.: Peroxynitrite and oxidative damage in experimental autoimmune uveitis. Invest. Ophthalmol. Vis. Sci. 1997, 38, 1333-1339.

36. Wystub S., Laufs T., Schmidt M., Burmester T., Maas U.: Localization of neuroglobin protein in the mouse brain. Neuroscience Lett. 2003, 346, 114-116.

37. Yang Y., Xia L., Xuejin G.: The distribution of neuroglobin in sheep retina. Chi. J. Anat. 2015, 38, 275-278.

38. Yang Y., Xia L., Guomin Z.: The distribution of neuroglobin in rabbit retina J. Histochem. Cytochem. 2015, 242, 139-144.

39. Yanxi G., Lulin W., Chaoyang W.: Exploration of the preparation method of bovine eye paraffin section. Chi. J. Dairy Cows. 2013, 171, 11-13 (in Chinese).

40. Yaoyu L., Hui-ling L., Tong T.: Immunohistochemistry study of the distribution of neuroglobin in the rat eyeball. Chi. Ophthal. Res. 2006, 245, 461-464

41. Ying Z.: Study on the changes of morphology, electrophysiology, and visual behavior of rat retina after ischemia [D]. J. Jinan Uni. 2013, 10, 113-118 (in Chinese).

42. Yong M., Yuanwei H., Zhiwu L.: Research advances in biological function and clinical application of neuroglobin. J. Clin. Med. 2016, 33, 104-105 (in Chinese).

43. Yu Z., Liu N., Wang Y.: Identification of neuroglobin-interacting proteins using yeast two-hybrid screening. Neuroscience 2012, 3, 99-105.

44. Zhenguo Y., Hongyun Y., Wenjuan D.: Effects of oxygen in the high altitude environment on the expression of retinal neuroglobin in rabbits. Adv. Ophthalmol. 2010, 30, 618-621.

45. Zhi L., Yixi L., Jinbo D.: Neuroprotective effects of neuroglobin on neurons J. Henan Uni. (Medical Sciences) 2016, 35, 225-228. (In Chinese).

46. Zhou L., Li Y., Yue B. Y.: Oxidative stress affects the cytoskeletal structure and cell-matrix interactions in cells from an ocular tissue: the trabecular meshwork. J. cell physiol. 1999, 180, 182-189, doi: 10.1002/(SICI)1097-4652 (199908)180.

Corresponding author: Liu Xia PhD, Anning District, Lanzhou City, Gansu Province, People's Republic of China; e-mail: drdugau2019@gmail.com 